\title{
Perfil sociodemográfico de portadores de Diabetes Mellitus e Hipertensão Arterial de uma comunidade religiosa
}

\section{Sociodemographic profile of patients with Diabetes Mellitus and Hypertension in a religious community}

Adriana Cristina Franco ${ }^{1}$, Eloisa Gabriela Linke ${ }^{2}$, Isabeli Lopes Kruk ${ }^{3}$, Julia Wolff Barretto $^{4}$, Juliana de Lima Germano ${ }^{5}$, Lívia Aro Rodrigues ${ }^{6}$

1. ORCID: https://orcid.org/0000-0002-3205-1780. Mestre. Faculdades Pequeno Príncipe, Curitiba-PR, Brasil. E-mail: francoadrianacristina@hotmail.com

2. ORCID: https://orcid.org/0000-0002-1647-6551. Acadêmica de Medicina. Faculdades Pequeno Príncipe, Curitiba-PR, Brasil. E-mail: elo26linke@gmail.com

3. ORCID: https://orcid.org/0000-0002-5198-042X. Acadêmica de Medicina. Faculdades Pequeno Príncipe, Curitiba-PR, Brasil. E-mail: isabelikruk@hotmail.com

4. ORCID: https://orcid.org/0000-0003-1784-419X. Acadêmica de Medicina. Faculdades Pequeno Príncipe, Curitiba-PR, Brasil. E-mail: juliawolffbarretto1@gmail.com

5. ORCID: https://orcid.org/0000-0002-3661-6937. Acadêmica de Medicina. Faculdades Pequeno Príncipe, Curitiba-PR, Brasil. E-mail: juliana.Igermano@gmail.com

6. ORCID: https://orcid.org/0000-0002-6506-3737. Mestrado. Universidade Federal do Paraná (UFPR), Programa de Pós-Graduação em Enfermagem. Curitiba, Paraná, Brasil. E-mail: alinebatista.abm@gmail.com

CONTATO: Autor correspondente: Adriana Cristina Franco | Endereço: Av Visconde de Guarapuava, n. 5395, Apto 31. Curitiba - Paraná. | Telefone: (41) 99983-0915 | E-mail: francoadrianacristina@hotmail.com

RESUMO Doenças crônicas não transmissíveis são um importante agravo da saúde da população mundial. Além de serem onerosas para o sistema público de saúde e um desafio para a gestão, carregam também um impacto negativo para a qualidade de vida do indivíduo. Este estudo trata-se de uma pesquisa 
exploratória-descritiva com abordagem quantitativa com o objetivo de conhecer o perfil sociodemográfico de portadores de Hipertensão Arterial Sistêmica e/ou Diabetes Mellitus em uma comunidade religiosa de Curitiba - PR. Foram entrevistados 100 portadores de HAS e/ou DM , que mostraram que os portadores das doenças possuíam idade avançada, maior nível de escolaridade, predomínio de caucasianos e distribuição semelhante entre os sexos, sendo que a maioria era portador de pelo menos mais uma comorbidade. Conclui-se que a influência das características locais no perfil epidemiológico das doenças revela a necessidade de medidas de prevenção e cuidado específicas para cada localidade, respeitando as suas particularidades.

DESCRITORES: Hipertensão. Diabetes Mellitus. Perfil de Saúde. Comorbidade.

ABSTRACT Non-communicable chronic diseases are an important health problem for the world population. In addition to being costly for the public health system and a challenge for management, they also have a negative impact on the individual's quality of life. This study is an exploratory-descriptive research with a quantitative approach in order to know the sociodemographic profile of patients with Systemic Arterial Hypertension and / or Diabetes Mellitus in a religious community in Curitiba - PR. One hundred patients with SAH and/or DM were interviewed, which showed that those with the disease had advanced age, higher education level, a predominance of Caucasians and a similar distribution between the sexes, with the majority having at least one other comorbidity. It is concluded that the influence of local characteristics on the epidemiological profile of diseases reveals the need for specific prevention and care measures for each location, respecting its particularities.

DESCRIPTORS: Hypertension. Diabetes Mellitus. Health Profile. Comorbidity. 


\section{INTRODUÇÃO}

oenças crônicas não transmissíveis tem um importante impacto na saúde da população mundial, inclusive da população brasileira. No Brasil, a Diabetes Mellitus e a Hipertensão Arterial Sistêmica juntos são os responsáveis pela primeira causa de mortalidade e internações no Sistema Único de Saúde ${ }^{1}$. No cenário mundial, de acordo com dados da Organização Mundial da Saúde, o número de portadores de Diabetes Mellitus e de Hipertensão Arterial Sistêmica tem aumentado ao longo do tempo, o que as tornam uma preocupação para a saúde pública². Ainda de acordo com esta, há uma associação entre a mortalidade prematura pelas doenças crônicas não transmissíveis e o nível de desenvolvimento socioeconômico dos países estudados, sendo que os locais onde há baixa e média renda para a população também enfrentam o maior risco de morte por este tipo de doença².

A Hipertensão Arterial Sistêmica caracteriza-se por ser uma condição clínica multifatorial, sendo que alguns fatores de risco como idade avança, sexo e etnia, excesso de peso, obesidade, consumo excessivo de sódio, etilismo crônico, sedentarismo e características genéticas são fatores de risco para o desenvolvimento da doença ${ }^{3}$. Do mesmo modo, a Diabetes Mellitus também é influenciada por fatores genéticos, biológico e ambientais ${ }^{4}$. Diante desse contexto, percebe-se que há um perfil do indivíduo mais prevalente que aumenta o risco para o desenvolvimento dessas doenças, onde um conjunto de fatores sociais, culturais, financeiros e genéticos contribuem para o desenvolvimento de doenças ${ }^{5}$.

Doenças crônicas não transmissíveis caracterizam-se por possuírem uma evolução lenta que muitas vezes não é percebida pelo portador até que a lesão no organismo esteja bastante avançada e os sintomas começam a tornar-se mais evidentes. Contudo, se houvessem medidas efetivas e ativas de prevenção de doenças e promoção de saúde através de hábitos saudáveis por parte dos sistemas de saúde e da população, tais condições poderiam ser tratadas precocemente, sendo revertidas ou melhoradas em seus prognósticos ${ }^{6}$. Ademais, tendo em vista a repercussão negativa do diagnóstico de uma doença crônica na qualidade de vida do indivíduo, o conhecimento do perfil do paciente contribui para a compreensão do seu contexto social e auxilia na adoção de medidas de controle efetivas da doença de forma individualizada ${ }^{7}$. Partindo dessa premissa, este estudo tem como objetivo definir o perfil de portadores de Hipertensão Arterial Sistêmica e portadores de Diabetes Mellitus de uma comunidade curitibana.

\section{MÉTODO}

Este trabalho tratou-se de uma pesquisa exploratória-descritiva com abordagem quantitativa para atingir o objetivo de conhecer o perfil sociodemográfico de portadores 
de Hipertensão Arterial Sistêmica e/ou Diabetes Mellitus. Caracteriza-se como um recorte do estudo original que incluiu aferição da pressão arterial, análise do perfil de hipertensos e diabéticos, hábitos de vida e percepção do processo de adoecimento, além do perfil epidemiológico dos entrevistados, tema este que é o enfoque deste artigo. O local de pesquisa selecionado foi uma comunidade religiosa católica Curitiba-PR. A igreja da comunidade possui mais de um século de existência, incluindo uma comunidade ativa e participativa em atividades religiosas, educacionais e sociais. Está localizado na região central da cidade, próximo a vários serviços de saúde e próximo a locais de grande circulação de pessoas. Assim, a instituição tem uma atuação importante em campanhas solidarias e também de apoio religioso para a comunidade próxima.

Está pesquisa envolveu um total de 100 participantes. Os critérios de inclusão adotados foram: mulheres e homens maiores de 18 anos, portadores de Hipertensão Arterial e/ou Diabetes Mellitus que aceitaram a participar deste estudo mediante a assinatura do Termo de Consentimento Livre e Esclarecido. Foram excluídos os participantes não pertencentes à comunidade escolhida. O local de pesquisa foi uma comunidade católica de Curitiba-Paraná que estimula o desenvolvimento de projetos sociais com enfoque na qualidade de vida da população. A abordagem destes foi feita antes e após as missas realizadas na instituição entre agosto e outubro de 2019.

O instrumento de pesquisa utilizado foi um questionário fechado aplicado na forma de entrevista. Os dados coletados incluíram identificação sociodemográfica, características específicas da apresentação da doença nos portadores, condições e hábitos de vida e a relação com processo de adoecimento crônico com enfoque na percepção do processo saúde-doença. Entende-se que os benefícios para os participantes foram refletir sobre o seu cuidado com a saúde e aprofundar seus conhecimentos sobre as características da sua doença, além de contribuir para o desenvolvimento de conhecimento científico. Os riscos inerentes a pesquisa envolveram a perda de anonimato e constrangimento durante a pesquisa, sendo que o pesquisadores comprometeram-se a usar dos dados sem nominá-los, garantindo ambiente adequado para a coleta de dados e conforto e orientações em caso de constrangimento ou dúvidas. Esta pesquisa foi aprovada pelo Comitê de Ética da Faculdade Pequeno Príncipe - FPP pelo parecer de número 3.424.334 em 26 de julho de 2019 , tendo respeitado todas os preceitos éticos previstos pela Resolução 466/12 do Conselho Nacional de Saúde.

A análise estatística foi feita a partir de gráficos e tabelas obtidos a partir do Excel, sendo que os resultados provenientes desta são apresentados junto à discussão neste artigo.

\section{RESULTADOS}

Esta pesquisa envolveu um total de 100 participantes, sendo 20 portadores de somente Hipertensão Arterial Sistêmica, 20 portadores de somente Diabetes Mellitus e 60 
portadores de ambas as doenças. Os dados foram organizados em planilhas do Excel e a análise permitiu a observação do perfil sociodemográfico deste grupo, sendo que as informações construídas são apresentadas abaixo. A análise é feita pela comparação de três subgrupos: 1. Portadores de somente Diabetes Mellitus; 2 . Portadores de somente Hipertensão Arterial Sistêmica e 3. Portadores de ambas as comorbidades.

Quanto ao gênero, houve predomínio da população masculina, com $51 \%$ do total de participantes. A população masculina foi maior entre os portadores somente de Diabetes Mellitus, com $60 \%$ destes, enquanto entre os portadores de ambas as doenças o número de mulheres foi maior, com $55 \%$ do total deste subgrupo. No subgrupo portador somente de Hipertensão Arterial, não houve predomínio de nenhum dos gêneros (Tabela 1).

Sobre à etnia, os caucasianos representam $85 \%$ dos participantes portadores apenas de Diabetes Mellitus, $86,7 \%$ dos participantes portadores apenas de hipertensão e $70 \%$ dos participantes portadores de ambas as doenças, totalizando $83 \%$ do total. Os pardos representam $15 \%$ dos pacientes portadores apenas de Diabetes Mellitus, $11 \%$ dos pacientes portadores apenas de hipertensão e $15 \%$ dos portadores de ambas as doenças, totalizando $13 \%$ do total. Só houve um participantes asiático, este portador de somente Hipertensão Arterial. A população negra representou $3 \%$ do total de participante, com $15 \%$ do subgrupo de portadores de apenas hipertensão.

Referente à escolaridade, participou desta pesquisa um analfabeto portador de somente diabetes, sem representantes nos outros subgrupos. Os participantes que concluíram o ensino primário representam $10 \%$ dos participantes portadores apenas de Diabetes Mellitus, $5 \%$ dos participantes portadores apenas de hipertensão e $15 \%$ dos participantes portadores de ambas as doenças, totalizando $8 \%$ dos participantes. Os participantes que concluíram o ensino fundamental representam $25 \%$ dos participantes portadores apenas de Diabetes Mellitus, $10 \%$ dos participantes portadores apenas de hipertensão e $5 \%$ dos participantes portadores de ambas as doenças, totalizando $12 \%$ dos participantes. Os participantes que concluíram o ensino médio representam $5 \%$ dos participantes portadores apenas de Diabetes Mellitus, 26,7\% dos participantes portadores apenas de hipertensão e $25 \%$ dos participantes portadores de ambas as doenças, totalizando $22 \%$ dos pacientes. Os participantes que concluíram ensino superior representam $55 \%$ dos participantes portadores apenas de Diabetes Mellitus, 58,3\% dos participantes portadores apenas de hipertensão e $55 \%$ dos participantes portadores de ambas as doenças, totalizando $57 \%$ dos participantes, sendo o nível de escolaridade predominante deste estudo (Tabela 1). 
Tabela 1. Perfil sociodemográfico dos participantes entrevistados

\begin{tabular}{|c|c|c|c|c|}
\hline \multicolumn{2}{|c|}{$\begin{array}{l}\text { Dado } \\
\text { sociodemográfico }\end{array}$} & $\begin{array}{l}\text { Portador de } \\
\text { somente DM }\end{array}$ & $\begin{array}{l}\text { Portador de } \\
\text { somente HAS }\end{array}$ & $\begin{array}{l}\text { Portador de } \\
\text { HAS e DM }\end{array}$ \\
\hline Valor & & $\mathrm{N}$ & $\mathrm{N}$ & $\mathrm{N}$ \\
\hline \multirow[t]{2}{*}{ Gênero } & Masculino & 12 & 30 & 9 \\
\hline & Feminino & 8 & 30 & 11 \\
\hline \multirow[t]{3}{*}{ Idade } & Média & 62,75 anos & 59,63 anos & 66,7 anos \\
\hline & Mínima & 42 anos & 18 anos & 43 anos \\
\hline & Máxima & 78 anos & 86 anos & 88 anos \\
\hline \multirow[t]{4}{*}{ Etnia } & Caucasiano & 17 & 52 & 14 \\
\hline & Pardo & 3 & 7 & 3 \\
\hline & Asiático & - & 1 & - \\
\hline & Negro & - & - & 3 \\
\hline \multirow[t]{5}{*}{ Escolaridade } & Analfabeto & 1 & - & - \\
\hline & Ensino Primário & 2 & 3 & 3 \\
\hline & Ensino Fundamental & 5 & 6 & 1 \\
\hline & Ensino Médio & 1 & 16 & 5 \\
\hline & Ensino Superior & 11 & 35 & 22 \\
\hline Total & & 20 & 60 & 20 \\
\hline
\end{tabular}

Legenda: HAS - Hipertensão Arterial Sistêmica; DM - Diabetes Mellitus.

Fonte: Elaborado pelos autores (2020).

A média de idade foi semelhante entre todos os grupos, sendo próxima à 60 anos. 0 subgrupo de hipertensos teve média de 59,63 anos, com mínimo de 18 anos e máximo de 86 anos. Quanto aos somente diabéticos, a média de idade foi de 62,75 anos, com mínimo de 42 e máximo de 78 anos. Já o subgrupo de portadores de ambas as doenças teve média de 66,7 anos, mínima de 43 e máxima de 48 anos (Tabela 2).

Tabela 2. Idade dos participantes entrevistados

\begin{tabular}{|c|c|c|c|c|}
\hline \multicolumn{2}{|c|}{$\begin{array}{l}\text { Dado } \\
\text { sociodemográfico }\end{array}$} & \multirow{2}{*}{$\begin{array}{l}\begin{array}{l}\text { Portador de } \\
\text { somente DM }\end{array} \\
62,75 \text { anos }\end{array}$} & \multirow{2}{*}{$\begin{array}{l}\begin{array}{l}\text { Portador de } \\
\text { somente HAS }\end{array} \\
59,63 \text { anos }\end{array}$} & \multirow{2}{*}{$\begin{array}{l}\text { Portador de } \\
\text { HAS e DM } \\
66,7 \text { anos }\end{array}$} \\
\hline Idade & Média & & & \\
\hline & Mínima & 42 anos & 18 anos & 43 anos \\
\hline & Máxima & 78 anos & 86 anos & 88 anos \\
\hline
\end{tabular}

Legenda: HAS - Hipertensão Arterial Sistêmica; DM - Diabetes Mellitus.

Fonte: Elaborado pelos autores (2020).

Outro ponto analisado foi a presença de comorbidades entre os participantes. Entre os entrevistados, $48 \%$ não possui nenhuma outra doença além de hipertensão e/ou diabetes. Foram relatadas 32 diferentes doenças, sendo que $52 \%$ dos entrevistados afirmaram possuir pelo menos uma comorbidade. As principais citadas foram dislipidemia, com 25 relatos $(25 \%)$, hipotireoidismo com 10 relatos (10\%), seguido por depressão $(5 \%)$, osteoporose (4\%) e pré-diabetes (4\%). O número máximo de doenças coexistentes en- 
contrada em um paciente desta pesquisa foi de cinco doenças. As doenças presentes nos participantes do estudo são apresentadas no gráfico abaixo (Gráfico 1).

Gráfico 1. Presença de comorbidade entre os participantes

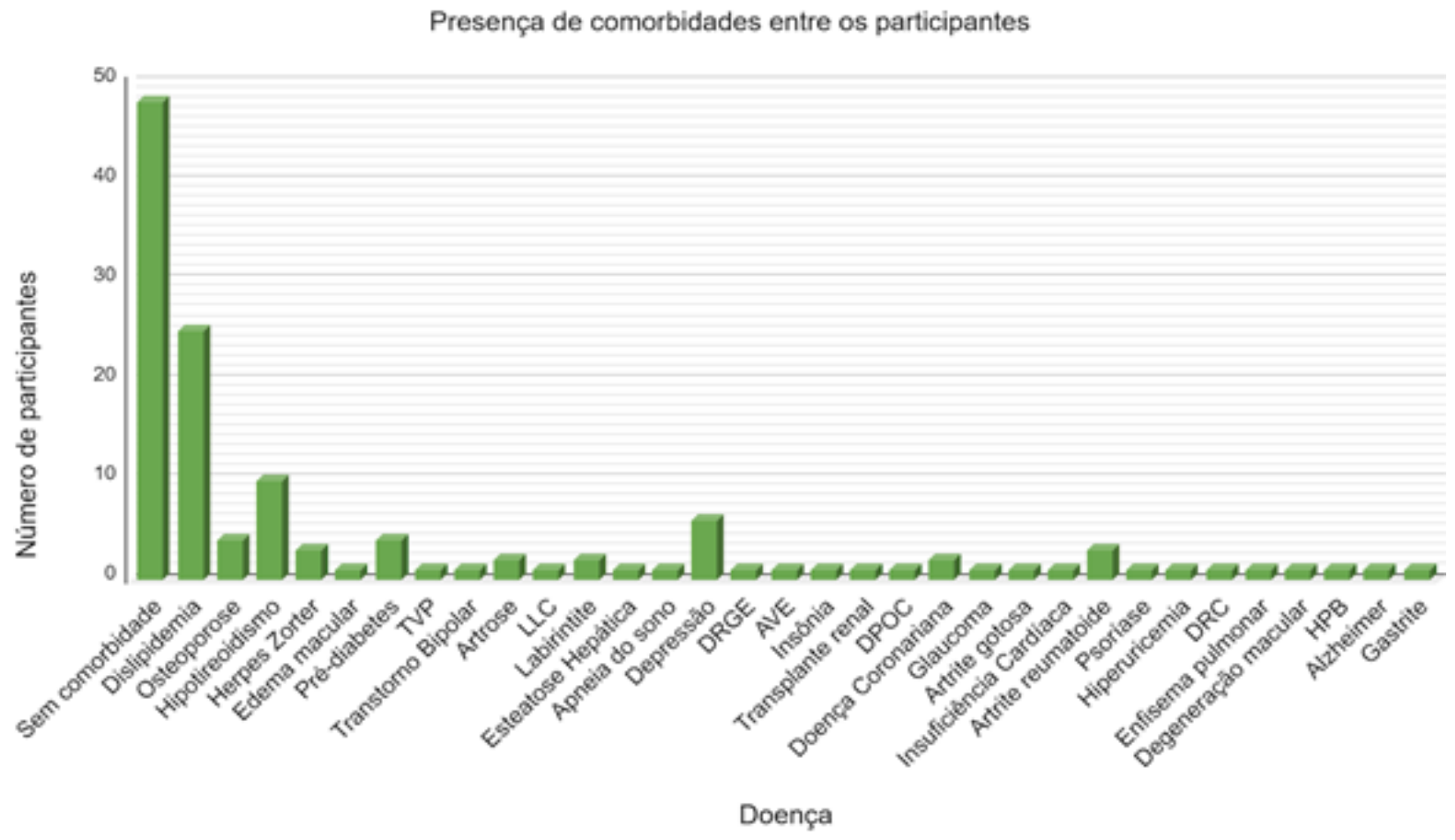

Legenda: TVP: Trombose Venosa Profunda; LLC: Leucemia Linfoide Crônica; DRGE: Doença do Refluxo Gastroesofágico; AVC: Acidente Vascular Cerebral; DPOC: Doença Obstrutiva Pulmonar Crônica; DRC: Doença Renal Crônica; HPB: Hiperplasia Prostática Benigna.

Fonte: Elaborado pelos autores, 2020.

A presença concomitante de mais de uma doença nos entrevistados foi maior entre os portadores de Hipertensão Arterial, com 63,33\% deste subgrupo. Em contrapartida, apenas $20 \%$ dos portadores de somente Diabetes Mellitus relataram possuir alguma comorbidade. Entre os portadores de ambas as doenças, houve a presença de pelo menos uma terceira doença associada em $55 \%$ dos casos. O presente estudo não avaliou a presença de sobrepeso e obesidade. Estes dados são apresentados na Tabela 3, que segue abaixo.

Tabela 3 - Comorbidades relatadas pelos participantes

\begin{tabular}{|c|c|c|c|c|c|c|}
\hline \multirow{2}{*}{$\begin{array}{l}\text { Presença de } \\
\text { Comorbidade } \\
\text { Valor }\end{array}$} & \multicolumn{2}{|c|}{$\begin{array}{l}\text { Portadores de } \\
\text { Somente DM }\end{array}$} & \multicolumn{2}{|c|}{$\begin{array}{l}\text { Portadores de HAS } \\
\text { e DM }\end{array}$} & \multicolumn{2}{|c|}{$\begin{array}{l}\text { Portadores de } \\
\text { somente HAS }\end{array}$} \\
\hline & $\mathrm{N}$ & $\%$ & $\mathrm{~N}$ & $\%$ & $\mathrm{~N}$ & $\%$ \\
\hline Sem comorbidade & 16 & $80 \%$ & 9 & $45 \%$ & 38 & $36,66 \%$ \\
\hline Com comorbidade & 4 & $20 \%$ & 11 & $55 \%$ & 22 & $63,33 \%$ \\
\hline
\end{tabular}

Legenda: N - Número; HAS - Hipertensão Arterial Sistêmica; DM - Diabetes Mellitus.

Fonte: Elaborado pelos autores (2020). 


\section{DISCUSSÃO}

A Diabetes Mellitus e a Hipertensão Arterial Sistêmica, juntos, são responsáveis pela primeira causa de mortalidade e de hospitalizações no Sistema Único de Saúde ${ }^{1,8}$. 0 diagnóstico de uma doença crônica dá início a uma nova jornada que traz uma mudança no estilo de vida que acompanhará o indivíduo por toda a sua vida, o que exigirá cuidados contínuos $^{9}$. O controle de níveis pressóricos e glicêmicos depende do tratamento permanente com adoção de medidas medicamentosas e não-medicamentosas, prevenindo complicações, aparecimento de comorbidades e mortalidade precoce ${ }^{10}$. Logo, tendo impacto sobre a qualidade de vida e a saúde dos indivíduos, a avaliação das características da população doente contribui para a adoção de medidas de controle efetivas para esta ${ }^{7}$.

Quanto ao perfil epidemiológico observado neste estudo, a maior prevalência de hipertensão na população masculina corrobora com o estudo anterior, visto que já se apontava a predominância maior no sexo masculino ao feminino ${ }^{11}$. Contudo, pesquisas apontam que a HAS é mais prevalente entre homens nas faixas etárias abaixo dos 50 anos e sofre inversão após, onde mulheres pós menopausa têm maior incidência da doença ${ }^{12}$. Outro estudo realizado em 2007 também evidenciou a feminização na velhice da hipertensão ${ }^{13}$. Tais estudos corroboram para o presente estudo, onde a média das idade de mulheres hipertensas foi de 65,3 anos e a de homens foi de 54,18 anos.

Estudos apontam que a hipertensão é uma doença que tem maior prevalência entre idosos que aumenta conforme o envelhecimento, visto que estão mais sujeitas a fatores de risco como excesso de peso, enrijecimento arterial e aumento da circunferência abdominal ${ }^{14}$. Essa prevalência aumentada da hipertensão na população idosa da comunidade abordada também pode ser evidenciada no presente estudo, onde a média de idade de portadores de diabetes, seja portador ou não de diabetes, foi maior que sessenta anos. De acordo com a literatura já disponível sobre o tema, há direta relação entre pressão arterial e idade, sendo que $60 \%$ dos indivíduos maiores de 65 anos podem apresentar Hipertensão Arterial Sistêmica ${ }^{15}$. Assim como a Hipertensão Arterial, a diabetes tipo 2 é uma doença que tem associação com o envelhecimento da população, sendo que $90 \%$ dos portadores desta doença são adultos ${ }^{5}$.

Os resultados deste estudo se contrapuseram a outros estudos prévios quanto a etnia, sendo que este apontada o predomínio de pessoas não brancas entre os portadores de $\mathrm{HAS}^{2}$. Pesquisas anteriores mostraram havia associação positiva entre a etnia negra e o número de portadores de doenças crônicas associada a uma maior propensão para o desenvolvimento da $\mathrm{HAS}^{16}$. Acredita-se que a divergência de outras pesquisas com análise epidemiológica, em que a prevalência de HAS é maior entre a população negra, deve-se a característica da população local, com significativa predominância de caucasianos. 
As diferenças socioeconômicas têm um papel importante na vida das pessoas podendo determinar as condições de saúde dessas pessoas, visto que aqueles com melhores condições têm maior acesso a informações, melhor entendimento da condição clínica e maior aderência ao tratamento. Logo se mostra taxas mais altas de doenças cardiovasculares em grupos com nível socioeconômico mais baixo ${ }^{17}$. Acredita-se que a maior proporção de participantes desta pesquisa com nível escolar mais elevado observado neste estudo está relacionado as características locais da comunidade estudada, onde o nível socioeconômico e educacional é mais elevado.

A presença de comorbidades clínicas compromete a evolução de pacientes, visto que as suas patologias se inter-relacionam e constituem fatores de risco entre si que podem agravar ainda mais o quadro do paciente ${ }^{18}$. Assim, e essencial que haja o acompanhamento do paciente de forma ampla voltada para todas as suas doenças e a sua relação com estas. A influência do processo do adoecimento pode acarretar no aparecimento de outras comorbidades, sendo que a preocupação e a ansiedade são fatores para a descompensação dos valores da pressão arterial e glicemia em portadores de Hipertensão Arterial e diabetes ${ }^{19}$. Assim, é essencial que haja o acompanhamento do paciente de forma ampla voltada para todas as suas doenças e a sua relação com o processo de adoecimento destas.

A presença de comorbidades interfere no tratamento da hipertensão, visto que está associado ao uso de múltiplos medicamentos, podendo sofrer interação medicamentosa e impactos em outras condições clínicas, principalmente no caso de pacientes idosos. A investigação de comorbidades em pacientes hipertensos também é importante devido a causas secundárias de hipertensão em alguns casos, como o hipotireoidismo. De acordo com a $7^{\circ}$ Diretriz de Hipertensão Arterial, esta pode ocorrer em cerca de $20 \%$ dos portadores de hipotireoidismo. Nesses casos o tratamento inicial para os pacientes é a reposição do hormônio tireoidiano, e hipertensivos apenas em casos refratários de acordo com Sociedade Brasileira de Cardiologia, o que demonstra a importância da investigação de doenças associadas de $2017^{20}$.

A dislipidemia é um dos principais fatores de risco associados ao desenvolvimento da aterosclerose e doença arterial coronariana. Os dados encontrados nesta pesquisa, com $25 \%$ dos entrevistados dislipidêmicos, são congruentes com os dados divulgados pelo Ministério da Saúde, através do sistema de Vigilância de Fatores de Risco e Proteção para doenças crônicas por inquérito telefônico (VIGITEL) com uma taxa de prevalência de 20,3\% para indivíduos maiores de 18 anos $^{21}$. Esta pesquisa também corrobora com outros estudos que evidenciaram a presença de $33,06 \%$ portadores de diabetes concomitantemente dislipidemia em portadores de Hipertensão Arterial ${ }^{22}$. A prevalência associada de hipertensão, diabetes e dislipidemia também fica evidente em outros estudos onde $22 \%$ dos portadores de diabetes apresentavam também hipercolesterolemia e 17\% Hipertensão Arterial23. 


\section{CONCLUSÃO}

A realização deste estudo permitiu conhecer o perfil epidemiológico da Hipertensão Arterial Sistêmica e da Diabetes Mellitus em uma comunidade local, assim como a relação dos portadores de tais doenças com o processo de saúde e doença. Foram observadas diferenças quantos aos dados sociodemográficos encontrados no grupo em questão e nos dados disponíveis na literatura sobre o tema. Conclui-se que a influência das características locais no perfil epidemiológico das doenças revela a necessidade de medidas de prevenção e cuidado específicas para cada localidade, respeitando as suas particularidades. Atribuímos os diferentes dados encontrados às características locais do campo de pesquisa escolhido e reforçamos a necessidade de replicação deste tipo de estudo em outros locais e grupos distintos, possibilitando a amplificação da amostra.

\section{REFERÊNCIAS}

1. SECRETARIA DE ESTADO DE SAÚDE DO PARANÁ. Linha guia de Diabetes Mellitus. 2. ed, Curitiba, 2018.

2. Neto JRG, Alves KKAF, Souza AKA, Alves MGL, Pessoa MAS, Almeida TCF, et al. Adesão terapêutica e qualidade de vida de hipertensos assistidos na atenção primária de saúde. Revista Nursing [Internet]. Fevereiro de 2019 [citado em 12 de dezembro de 2020]; 22 (249), 2600-2603. doi: http://dx.doi.org/10.36489/ nursing.2019v22i249p2598-2603

3. SOCIEDADE BRASILEIRA DE CARDIOLOGIA. VI Diretrizes Brasileiras de Hipertensão, Conceituação, Epidemiologia e Prevenção Primária. Rio de Janeiro, 2010.

4. SOCIEDADE BRASILEIRA DE DIABETES. Controle da glicemia no paciente hospitalizado. São Paulo, 2015.

5. Fontana S, Araújo AC. Os processos educativos no contexto das práticas de saúde em Unidades Básicas de Saúde e Unidades de Saúde da Família. CuidArte Enferm [Internet]. Junho de 2018 [citado em 12 de dezembro de 2020], 12 (1): 81-91. Disponível em: http://www.webfipa.net/facfipa/ner/sumarios/cuidarte/2018v1/81.pdf

6. Gualano B, Tinucci T. T. Sedentarismo, exercício físico e doenças crônicas. Rev. Bras. Educ. Fís. Esporte [internet]. 2011 [citado em 30 de novembro de 2020]; 25. doi: https://doi.org/10.1590/S180755092011000500005

7. Oliveira-Campos M, Rodrigues-Neto JF, Silveira MF, Neves DMR, Vilhena JM, Oliveira JF, Magalhães JC, Drumond D. Impacto dos fatores de risco para doenças crônicas não transmissíveis na qualidade de vida. Ciência \& Saúde Coletiva [Internet]. Janeiro de 2013 [citado em 12 de dezembro de 2020] 18(3): 873882. doi: http://dx.doi.org/10.1590/S1413-81232013000300033

8. Buccolini, CS. Morbidade por doenças crônicas no brasil: situação atual e futura. $1^{\text {a }}$ Edição. Rio de Janeiro: Fundação Oswaldo Cruz, 2016.

9. Silva JVM, Mantovani MF, Kalinke LP, Ulbrich EM. Hypertension and Diabetes Mellitus Program evaluation on user's view. Rev Bras Enferm [Internet]. Agosto de 2015 [citado em 12 de dezembro de 2020]; 68(4): 626-32. doi: https://doi.org/10.1590/0034-7167.2015680408i

10. Mantovani MF, Mendes FRP, Ulbrich EM, Bandeira JM, Fusuma F, Gaio DM. As representações dos usuários sobre a doença crônica e a prática educativa. Rev. Gaúcha Enferm. [Internet]. Dezembro de 2011 [citado em 12 de dezembro de 2020] ; 32( 4 ): 626-668. doi: https://doi.org/10.1590/S198314472011000400004 
11. Silva EC, Soares MMSA, Guimarães LV, Segri NJ, Lopes MA, Espinosa MM. Prevalência de Hipertensão Arterial Sistêmica e fatores associados em homens e mulheres residentes em municípios da Amazônia Legal. Rev. Bras. Epidemiol.,[Internet]. 2016 Mar [citado em 12 de dezembro de 2020]; 19 (1): 38-51. doi: https://doi.org/10.1590/1980-5497201600010004

12. Dantas RCO, Roncalli AG. Protocolo para indivíduos hipertensos assistidos na Atenção Básica em Saúde. Ciênc. saúde coletiva [Internet]. Janeiro de 2019 [citado em 12 de dezembro de 2020]; 24 (1): 295306. doi: https://doi.org/10.1590/1413-81232018241.35362016

13. Stipp MAC, Leite JL, Cunha NM, Assis LS, Andrade MP, Simões RD. O consumo do álcool e as doenças cardiovasculares: uma análise sob o olhar da enfermagem. Esc. Anna Nery [Internet]. Dezembro de 2007 [citado em 12 de dezembro de 2020]; 11( 4 ): 581-585. doi: https://doi.org/10.1590/S141481452007000400004

14. Bezerra ALL, Bezerra D, Pinto DS, Bonzi ARB, Pontes RMN, Velosi JAP. Perfil Epidemiológico de idosos hipertensos no brasil: uma revisão integrativa. Rev Med [Internt]. Fevereiro de 2018 [citado em 12 de dezembro de 2020]; 97(1):103-7. doi: https://doi.org/10.11606/issn.1679-9836.v97i1p103-107

15. Gomes TJO, Rocha MV, Santos AA. Controle da pressão arterial em pacientes atendidos pelo programa Hiperdia em uma Unidade de Saúde da Família. Rev. Bras. Hipertens [Internet]. Setembro de 2010 [citado em 20 de dezembro de 2020]; 17 (3), 132-139. Disponível em: http://departamentos.cardiol.br/dha/ revista/17-3/03-controle.pdf

16. Sousa ALL, Batista SR, Sousa AC, Pacheco JAS, Vitorino PVO, Pagotto V. Prevalência, tratamento e controle da hipertensão em idosos em uma capital brasileira. Arq. Bras. Cardiol. [Internet]. Março de 2019 [citado em 12 de dezembro de 2020]; 112 (3): 271-278. doi: https://doi.org/10.5935/abc.20180274

17. Cipullo JP, Martin JFV, Ciorlia LAS, Godoy MRP, Cação JC, Loureiro AAC et al . Prevalência e fatores de risco para hipertensão em uma população urbana brasileira. Arq. Bras. Cardiol. [Internet]. Abril de 2010 [citado em 12 de dezembro de 2020] ; 94( 4 ): 519-526. doi: https://doi.org/10.1590/S0066-782X2010005000014

18. Chaves AEA, Morais GA, Gurgel KOB, Reis MVC, Geraldo PFT, Quintão TMG. Avaliação do autoconhecimento sobre comorbidades em pacientes tratados ambulatoriamente. Rev. Med. Minas Gerais [Internet]. 2018 [citado em 12 de dezembro de 2020]; vol, 28 (4): 19-26. Disponível em: http://rmmg.org/ exportar-pdf/2303/v28s4a04.pdf

19. Pereira NPA, Lanza FM, Viegas SM. Vidas em tratamento para Hipertensão Arterial Sistêmica e Diabetes Mellitus: sentimentos e comportamentos. Rev. Bras. Enferm. [Internet]. Fevereiro de 2019 [citado em 12 de dezembro de 2020] ; 72( 1 ): 102-110. doi: https://doi.org/10.1590/0034-7167-2016-0500

20. Malachias MVB. et al . $7^{\text {a }}$ Diretriz Brasileira de Hipertensão Arterial: Capítulo 1 - Conceituação, Epidemiologia e Prevenção Primária. Arq. Bras. Cardiol. 2016 Set, 107 (3), 1-6.

21. Almeida AV et al. A Feminização da Velhice: em foco as características socioeconômicas, pessoais e familiares das idosas e o risco social. Revista Textos \& Contextos [Internet] 2015. [acesso em 2020 nov 30]; 14(1). Disponível em: https://www.redalyc.org/articulo.oa?id=321540660010

22. Freitas LC, Rodrigues GM, Araújo FC, Falcon EBS, Xavier NF, Lemos ELC, Pires CAA. Perfil dos hipertensos da Unidade de Saúde da família Cidade Nova, município de Ananindeua-PA. Rev Bras Med Fam Comunidade [Internet]. Março de 2012 [citado em 12 de dezembro de 2020]; 7(22): 13-9. doi: https://doi. org/10.5712/rbmfc7(22)288

23. Freitas LRS, Garcia LP. Evolução da prevalência do diabetes e deste associado à Hipertensão Arterial no Brasil: análise da Pesquisa Nacional por Amostra de Domicílios, 1998, 2003 e 2008. Epidemiol. Serv. Saúde [Internet]. 2012 Mar [citado 2020 Dez 12] ; 21( 1 ): 07-19. Disponível em: http://scielo.iec.gov.br/ scielo.php?script=sci_arttext\&pid=S1679-49742012000100002 